\title{
Theophylline improves lipopolysaccharide-induced alveolarization arrest through inflammatory regulation
}

\author{
HUA HE $^{1 *}$, FEI CHEN $^{1 *}$, WENSI NI $^{1}$, JIANHUI LI $^{1}$ and YONGJUN ZHANG ${ }^{1,2}$ \\ ${ }^{1}$ Neonatology Department, Xinhua Hospital, Shanghai Jiaotong University School of Medicine, Shanghai 200092; \\ ${ }^{2}$ MOE and Shanghai Key Laboratory of Children's Environmental Health, Shanghai 200092, P.R. China
}

Received August 6, 2013; Accepted March 11, 2014

DOI: $10.3892 / \mathrm{mmr} .2014 .2188$

\begin{abstract}
Bronchopulmonary dysplasia (BPD) is characterized by alveolar simplification with decreased numbers of alveoli and increased airspace. BPD, frequently suffered by very low birth weight infants, has been closely associated with intrauterine infection. However, the underlying mechanisms of BPD remain unclear. In the present study, it was identified that administration of intra-amniotic lipopolysaccharide (LPS) to pregnant rats on embryonal day 16.5 (E16.5) induced significant alveolarization arrest similar to that of BPD in neonatal pups, and theophylline injected subcutaneously into the newborns improved the pathological changes. To further investigate the underlying mechanism of the morphogenesis amelioration of theophylline, cytokine antibody arrays were performed with the lung lysates of neonatal rats. The results indicated that LPS upregulated a series of pro-inflammatory cytokines and theophylline significantly attenuated the expression levels of pro-inflammatory cytokines tumor necrosis factor- $\alpha$, macrophage inflammatory protein (MIP)- $1 \alpha$ and MIP-2, and markedly elevated the production of tumor growth factor (TGF)- $\beta$ family members TGF- $\beta 1$, TGF- $\beta 2$ and TGF- $\beta 3$, which are anti-inflammatory cytokines. Accordingly, it was hypothesized that theophylline may protect against BPD and improve chorioamnionitis-induced alveolar arrest by regulating the balance between pro-and anti-inflammatory cytokine expression.
\end{abstract}

\section{Introduction}

Bronchopulmonary dysplasia (BPD) is the most common chronic lung disease in preterm infants (1). In recent years, with the development of perinatal medicine, the survival rate of

Correspondence to: Dr Yongjun Zhang, Neonatology Department, Xinhua Hospital, Shanghai Jiaotong University School of Medicine, 1665 Kongjiang Road, Shanghai 200092, P.R. China

E-mail: zhangyongjun@sjtu.edu.cn

*Contributed equally

Key words: bronchopulmonary dysplasia, theophylline, chorioamnionitis, cytokine antibody array very low birth weight infants (VLBWI) has been continuously improving, however, the incidence of BPD is gradually on the rise and the disease exhibits a number of novel features. Infant patients initially have minimal or absent signs of respiratory distress syndrome but subsequently develop oxygen dependency and require a ventilator within the first two weeks of life, which may last for weeks and months, termed 'new BPD'. A large number of these infants may have been exposed to an intrauterine inflammatory environment, such as chorioamnionitis, and are subsequently born with inflammation of the lungs. Following birth, various risk factors, including pulmonary or systemic infections, high concentrations of oxygen inspiration and mechanical ventilation, may act synergistically, amplifying the inflammation and subsequently affecting normal alveolarization in preterm infants (2). In view of the central role of inflammation in the development of BPD and the known anti-inflammatory properties of corticosteroids, these drugs have been used in the prevention and treatment of BPD for decades. However, a number of studies have demonstrated that postnatal corticosteroid treatment, mainly with dexamethasone in relatively high doses, may result in long-term adverse neurocognitive outcomes, brain growth reduction and hippocampus degeneration $(3,4)$. Therefore, the present study aimed to identify the efficacy of another clinical drug to suppress the effects of chorioamnionitis on neonatal lung development with minimal side effects.

Theophylline, a classical bronchodilator drug for the treatment of chronic lung diseases, including asthma and chronic obstructive pulmonary disease (COPD), inhibits phosphodiesterase (PDEs) activity, antagonizes adenosine receptors and increases intracellular cyclic adenosine monophosphate (cAMP) levels (5). Theophylline at high concentrations is associated with numerous side effects, including headache and nausea mediated by PDE inhibition, and cardiac arrhythmias and seizures due to adenosine receptor antagonism (5). Therefore, the clinical use of theophylline for the treatment of respiratory diseases has been rapidly declining. Previously, numerous studies have demonstrated that physiological doses of theophylline have anti-inflammatory effects by inhibiting inflammatory factors and increasing anti-inflammatory cytokine secretion to suppress the development of inflammatory responses $(2,6)$. Our previous studies have demonstrated that theophylline restored histone deacetylase (HDAC) activity to inhibit inflammatory factor IL-8 expression and improve 
A

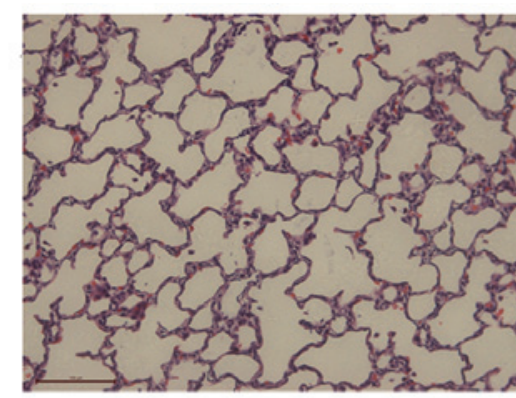

saline

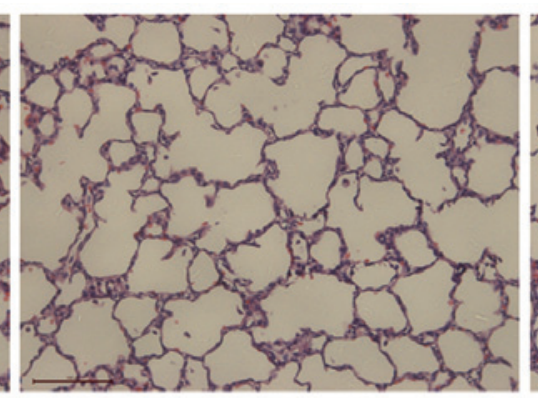

LPS

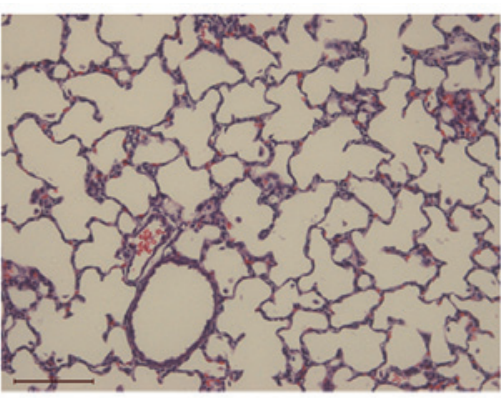

LPS+theo
B

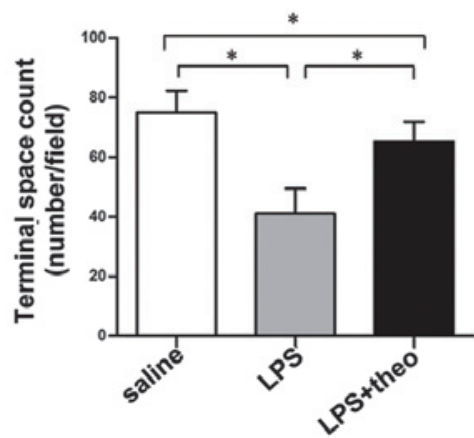

C

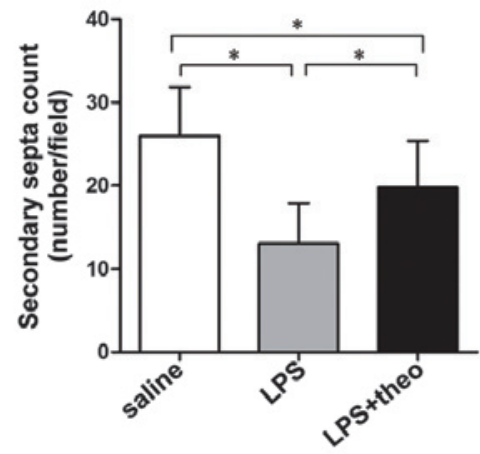

Figure 1. Theophylline improves alveolarization in newborn rats with prenatal intra-amniotic LPS injection. (A) The representative photomicrographs of pup lung sections (hematoxylin and eosin stains; magnification, x200; scale bar, $100 \mu \mathrm{m}$ ) at P7. (B) The rat pups in LPS+theo group (pups injected with LPS+theophylline) had significantly more terminal space counts than the LPS group. $\mathrm{n}=25$ random non-overlapping fields from five lungs/group. The experiment was repeated three times independently. (C) The secondary septa numbers were significantly increased in the LPS+theo group than the LPS group. $\mathrm{n}=25$ random non-overlapping fields from five lungs/group. The experiment was repeated three times independently. ${ }^{*} \mathrm{P}<0.05$. P7, postnatal day 7 ; LPS, lipopolysaccharide.

alveolar development in the lungs of neonatal rats induced by hyperoxia (7). Therefore, it was hypothesized that theophylline may also ameliorate the pathophysiological changes in BPD induced by chorioamnionitis through inflammatory regulation.

LPS is a potent pro-inflammatory stimulating agent associated with intrauterine inflammatory processes, including chorioamnionitis (1,2). Intra-amniotic LPS administration in pregnant rodents may mimic BPD in human premature infants, resulting in lung development inhibition, fewer alveoli and secondary septa in the lungs (8). In the present study, LPS was injected into the amniotic cavity of Sprague-Dawley (SD) pregnant rats (E16.5) and theophylline was administered into neonatal rats to examine the hypothesis that theophylline may restore the inflammatory equilibrium in the lungs of neonatal rats exposed to chorioamnionitis, to improve disrupted alveolarization in BPD.

\section{Materials and methods}

Intra-amniotic LPS administration. Pregnant rats on embryonal day 16.5 (E16.5) that were used in the present study were purchased from the Shanghai Laboratory Animal Center (Shanghai, China). The experimental procedures involving rats were performed under the guidelines and permission of the Experts Committee of Laboratory Animal Sciences of the Shanghai Jiaotong University School of Medicine (Shanghai, China). On gestation day 16.5, gravid females were anesthetized with an intraperitoneal injection of $10 \%$ chloral hydrate at a dose of $3.5 \mathrm{ml} / \mathrm{kg}$ body weight. Following midline abdominal section, $1 \mu \mathrm{g}$ lipopolysaccharide (LPS; 055: B5, Sigma-Aldrich, St. Louis, MO, USA) at a concentration of $0.2 \mu \mathrm{g} / \mu \mathrm{l}$, solubilized in saline, was injected into the amniotic sac of each fetus. The same volume of sterile, endotoxin-free saline without LPS was injected in the saline control groups. The pups were delivered spontaneously 3-4 days following these injections.

Theophylline drug administration. Following delivery, the pups of the same litter with intra-amniotic LPS injection were randomly divided into two groups: The LPS+theo group and the LPS group. In the LPS+theo group pups were injected with theophylline (20 mg/kg/day; Sigma, St. Louis, MO, USA) and the LPS group were treated with the saline of the same volume. Theophylline was dissolved in saline to a final concentration of $5 \mathrm{mg} / \mathrm{ml}$ and injected subcutaneously into the neck of the pups, first within $6 \mathrm{~h}$ after birth and then once a day until postnatal day 7 (P7). Pups of all groups obtained identical postnatal care following drug administration.

Lung processing. At $\mathrm{P} 7$, all of the surviving rat pups were sacrificed by an intraperitoneal injection of $10 \%$ chloral hydrate. The lung and trachea were exposed by thoracotomy, and the right bronchus and trachea were ligated. The pups then received an intratracheal instillation of buffered formaldehyde (4\% paraformaldehyde solubilized in PBS; $\mathrm{pH} 7.4$ ) at a pressure of $20 \mathrm{~cm} \mathrm{H}_{2} \mathrm{O}$ for $20 \mathrm{~min}$. For histological analysis, the left lung was further fixed in buffered formal- 
dehyde for $24 \mathrm{~h}$ at $4^{\circ} \mathrm{C}$, and followed by hematoxylin and eosin staining. For the antibody array analysis, the right lung without perfusion was excised, frozen in liquid nitrogen and stored at $-80^{\circ} \mathrm{C}$.

Lung morphometry. Five pups were selected in each group and five random non-overlapping fields in one distal lung section per pup were utilized for the morphometric examinations. The terminal air spaces and alveolar secondary septa were counted manually at a magnification of x200.

Cytokine antibody array. Protein extraction from the four right lungs of the saline group, LPS group and LPS+theo group were pooled respectively for one antibody array (RayBio ${ }^{\circledR}$ Biotin Label-based Rat Antibody Array I; RayBiotech, Norcross, GA, USA), including 90 cytokines. Briefly, $2 \mathrm{ml}$ of $1 \mathrm{X}$ blocking buffer was added to the array membranes and incubated at room temperature for $30 \mathrm{~min}$. The blocked membranes were then incubated with $1 \mathrm{ml}$ protein extraction of pooled lung tissues at room temperature for $1-2 \mathrm{~h}$. Thereafter, the membranes were washed three times with $2 \mathrm{ml}$ of $1 \mathrm{X}$ wash buffer I and twice with $2 \mathrm{ml}$ of $1 \mathrm{X}$ wash buffer II (RayBiotech). Following this, $1 \mathrm{ml}$ diluted biotin-conjugated anti-cytokines antibodies (RayBiotech) against cytokines extracted from samples, which were captured by the antibodies fixed in the cytokine antibody arrays, were added to each membrane and incubated at room temperature for 1-2 h. Membranes were washed once more as mentioned previously and then each incubated with $2 \mathrm{ml}$ of 1,000-fold diluted horseradish peroxidase-conjugated streptavidin at room temperature for $2 \mathrm{~h}$. The intensities of the signals were quantified by densitometric analysis. The internal positive controls of each membrane were used to normalize the relative expression levels of 90 cytokines from the different membranes being compared.

Statistical analysis. The results are expressed as the mean \pm standard deviation. Statistical analysis was performed with one-way analysis of variance followed by Student-Newman-Keuls test for multiple comparisons using SPSS 18.0 (IBM, Armonk, NY, USA). P<0.05 was considered to indicate a statistically significant difference. In the cytokine antibody array detection, an expression alteration of $\geq 2$-fold was considered to be significant.

\section{Results}

Theophylline improves alveolarization in newborn rats with intra-amniotic LPS injection. Gravid rats were injected with LPS or saline into the amniotic sacs at E16.5. Histological analysis revealed marked differences in the lung structure of neonatal rats in the three groups (Fig. 1A). Pups at 7 days-old in the LPS group demonstrated notably fewer terminal spaces and secondary septa than the saline group $(41 \pm 8$ vs. $75 \pm 7$, $13 \pm 5$ vs. $26 \pm 6$, respectively), suggesting successful induction of rodent BPD by LPS. Furthermore, it was identified that the terminal air space count and secondary septa number in the LPS+theo group increased significantly compared with the LPS group ( $65 \pm 7$ vs. $41 \pm 8,20 \pm 6$ vs. $13 \pm 5$, respectively), although these parameters did not completely return to the levels of the saline group (Fig. 1B and C). These data suggest
Table I. Cytokine expression upregulated by $\geq 2$-fold in the LPS group compared with the saline group.

\begin{tabular}{lccr}
\hline Cytokine & LPS & Saline & Fold change \\
\hline 乃-Catenin & 0.0652770 & 0.0206126 & 3.1668524 \\
b-FGF & 0.1367899 & 0.0196013 & 6.9786044 \\
CCR4 & 0.0931056 & 0.0444458 & 2.0948132 \\
CINC-3 & 0.0955325 & 0.0273635 & 3.4912371 \\
CNTF & 0.1087187 & 0.0034210 & 31.7798012 \\
CNTF R $\alpha$ & 0.0898292 & 0.0010705 & 83.9133115 \\
CXCR4 & 0.2192642 & 0.0165675 & 13.2345979 \\
EGFR & 0.1818090 & 0.0226351 & 8.0321594 \\
E-Selectin & 0.1881998 & 0.0601068 & 3.1310900 \\
FADD & 0.1466997 & 0.0676230 & 2.1693774 \\
IFN- $\gamma$ & 0.1696340 & 0.0206126 & 8.2296299 \\
IL-1 $\alpha$ & 0.0958965 & 0.0364103 & 2.6337766 \\
IL-1 $\beta$ & 0.3738986 & 0.0083954 & 44.5363370 \\
IL-1 R6/IL-1 R rp2 & 0.2595509 & 0.0738272 & 3.5156518 \\
IL-2 & 0.1620297 & 0.0789109 & 2.0533247 \\
IL-6 & 0.3586091 & 0.0986990 & 3.6333606 \\
IL-10 & 0.3855882 & 0.1265773 & 3.0462677 \\
IL-12/IL-23 p40 & 0.4204143 & 0.0962392 & 4.3684326 \\
IL-13 & 0.3703392 & 0.1019515 & 3.6325041 \\
Leptin (OB) & 0.2086668 & 0.0723787 & 2.8829868 \\
NGFR & 0.2634339 & 0.1211929 & 2.1736741 \\
Orexin A & 0.2017905 & 0.0795122 & 2.5378554 \\
Osteopotin/SPP1 & 0.2419963 & 0.1153440 & 2.0980401 \\
RALT/MIG-6 & 0.3737368 & 0.1330002 & 2.8100473 \\
RELM $\beta$ & 0.4076326 & 0.1191157 & 3.4221561 \\
Resistin & 0.3374141 & 0.1454634 & 2.3195808 \\
TIE-2 & 0.1393786 & 0.0640699 & 2.1754157 \\
TIMP-3 & 0.0981212 & 0.0029564 & 33.1894196 \\
\hline & & & \\
\hline
\end{tabular}

b-FGF, basic fibroblast growth factor; CCR4, chemokine receptor type 4; CINC-3, cytokine-induced neutrophil chemoattractant 3; CNTF, ciliary neurotrophic factor; CXCR, C-X-C chemokine receptor type 4; EGFR, epidermal growth factor receptor; FADD, Fasassociated protein with death domain; IFN, interferon; IL, interleukin; NGFR, nerve growth factor receptor; SPP1, secreted phosphoprotein 1; RALT/MIG-6, receptor-associated late transducer/mitogen inducible gene-6; RELM $\beta$, resistin-like molecule $\beta$; TIE- 2 , tyrosine kinase with immunoglobulin-like and EGF-like domains 2; TIMP, tissue inhibitor of metalloproteinase.

that theophylline may improve the lung development/function and restore alveolarization in LPS-induced rodent BPD.

LPS increases the expression levels of pro-inflammatory cytokines. In the present study, 90 cytokines in the lungs of the rats in the saline and LPS groups were examined by cytokine antibody array (Fig. 2A and B), in which the levels of 28 cytokines had changed significantly ( $>2$-fold; Table I). It is well-established that LPS is a potent agent that induces the expression of pro-inflammatory cytokines in various systems (1,2). Among the upregulated factors, a series of pro-inflammatory cytokines, including interleukin (IL)-1 $\alpha$, 
A
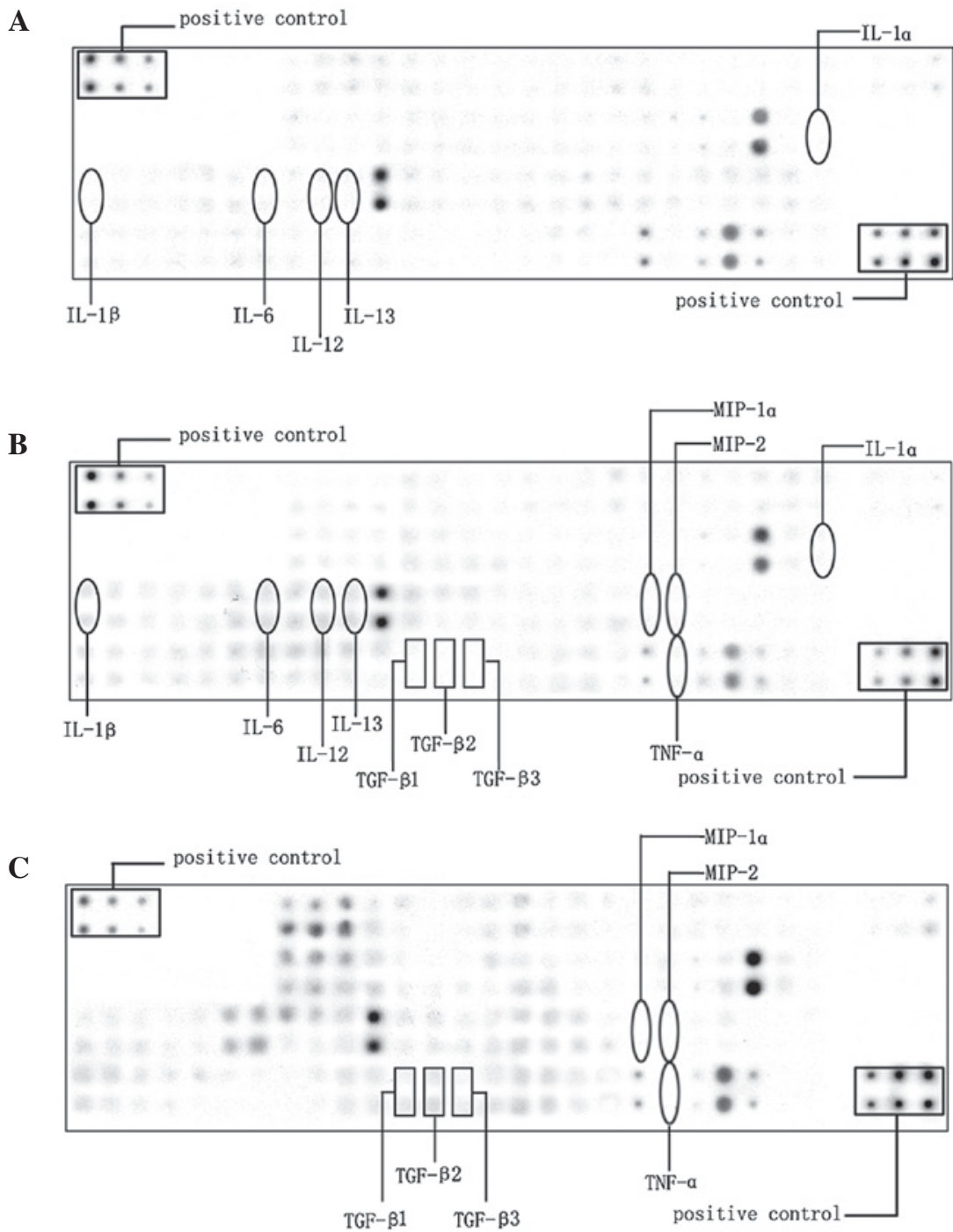

Figure 2. Expression levels of 90 cytokines in lungs of pups at P7 were assayed using the RayBio® Biotin Label-based Rat Antibody Array I. (A) The saline group, (B) LPS group and (C) LPS+theo group (pups injected with LPS+theophylline). Ellipse represents markedly altered pro-inflammatory cytokines, vertical square represents significantly changed anti-inflammatory cytokines and horizontal square represents the positive control. P7, postnatal day 7; LPS. lipopolysaccharide.

IL-1 $\beta$, IL-6, IL-12 and IL-13 were increased by 2.6, 44.5, 3.6, 4.4 and 3.6 times, respectively.

Theophylline regulates inflammatory balance. To examine the hypothesis that theophylline treatment prevents LPS-induced inflammation, cytokine antibody arrays of the lung samples from the LPS+theo group were performed and compared with those of the LPS group (Fig. 2B and C). It was identified that the expression levels of 36 cytokines altered significantly in the LPS+theo group as compared with the LPS group, with 20 cytokines upregulated (Table II) and 16 cytokines downregulated (Table III). A larger number of the cytokines were considered to be associated with inflammation. In particular, the pro-inflammatory cytokines tumor necrosis factor (TNF)- $\alpha$, macrophage inflammatory protein (MIP)- $1 \alpha$ and MIP-2 were significantly decreased $37.4,12.5$ and 8.6 times, respectively (Table III) and the anti-inflammatory cytokine tumor growth factor (TGF)- $\beta$ family members TGF- $\beta 1$, TGF- $\beta 2$ and TGF- $\beta 3$ were elevated $2.4,56.9$ and 17.8 times, respectively (Table II). These data suggest that theophylline may reduce the inflammatory responses induced by LPS and therefore improve the alveolarization in the rodent BPD lung.

\section{Discussion}

Human lung development is a complex process, which is characterized by five stages of development based on histological appearance, including embryonic, pseudoglandular, canalicular, saccular and alveolar stages (9). Premature infants delivered at 26-36 weeks of gestation are born in the saccular phase of lung development and are particularly susceptible to BPD. Disrupted saccular phase development results in pulmonary interstitium remolding and morphological changes in preterm infants with BPD (10). Lung development during the saccular phase involves a number of vital morphological processes, including growth and widening of distal airways, further division of the distal airspaces by primary septation, thinning of the air-blood barrier and formation of the double capillary network (11). Therefore, the saccular phase has a key role in preparing the distal lung for the subsequent formation of alveoli and gas exchange at birth. 
Table II. Cytokine expression upregulated by $\geq 2$-fold in the LPS+theo group compared with the LPS group.

\begin{tabular}{lccr}
\hline Cytokine & LPS+theo & LPS & Fold change \\
\hline TGF- $\beta 2$ & 0.3311682 & 0.0058178 & 56.9229390 \\
Thrombospondin & 0.2600238 & 0.0116020 & 22.4119807 \\
TGF- $\beta 3$ & 0.1512473 & 0.0084874 & 17.8202160 \\
ACTH & 0.9745741 & 0.0705353 & 13.8168279 \\
ADFP & 1.0446070 & 0.1205296 & 8.6668081 \\
Activin A & 0.7720283 & 0.1109029 & 6.9613009 \\
ICK & 0.2347178 & 0.0435158 & 5.3938547 \\
GFR $\alpha-1$ & 0.2692111 & 0.0643467 & 4.1837572 \\
MuSK & 0.0252079 & 0.0060605 & 4.1593573 \\
E-Selectin & 0.7515612 & 0.1881998 & 3.9934209 \\
FADD & 0.5765773 & 0.1466997 & 3.9303222 \\
L-Selectin/CD62L & 0.4030973 & 0.1185881 & 3.3991385 \\
Hepassocin & 0.1511165 & 0.0447292 & 3.3784738 \\
TIE-2 & 0.4536439 & 0.1393786 & 3.2547603 \\
$\beta$-Catenin & 0.2105234 & 0.0652770 & 3.2250766 \\
EG-VEGF/PK1 & 0.6236254 & 0.2039343 & 3.0579726 \\
GFR $\alpha-2$ & 0.2219340 & 0.0861484 & 2.5761818 \\
TGF- $\beta 1$ & 0.2109158 & 0.0885753 & 2.3812022 \\
TIMP-1 & 0.3927330 & 0.1782495 & 2.2032764 \\
$\beta$ 3-NGF & 0.1306167 & 0.0624861 & 2.0903328 \\
\hline TGF, tumor growth & factor; ACTH, adenocorticotropic hormone; \\
ADFP, adipose deifferentiation-related protein; ICK, intestinal cell \\
(MAK-like) kinase; GFR, growth factor receptor; MuSK, muscle- \\
specific kinase receptor; FADD, Fas-associated protein with death \\
domain; TIE-2, tyrosine kinase with immunoglobulin-like and \\
EGF-like domains 2; EG-VEGF/PK1, endocrine gland-derived vas- \\
cular endothelial growth factor/prokineticin 1; TIMP, tissue inhibitor \\
of metalloproteinase NGF, nerve growth factor. & \\
\hline & & & \\
\hline
\end{tabular}

In the present study, an intra-amniotic injection of LPS was administered to pregnant SD rats at E16.5, a transitional period from the canalicular to saccular stage. Newborn rats exposed in utero to LPS demonstrated arrested lung development, characterized by fewer numbers of alveoli and secondary septa counts. The results unexpectedly revealed that theophylline improved alveolarization and partly ameliorated the process of alveolar arrest. These data suggest the potential benefits of theophylline in the treatment of BPD infants; however, elucidating the mechanisms underlying these effects requires further study. In the present study, the antibody cytokine arrays demonstrated that a series of pro-inflammatory cytokines were upregulated in the LPS group, as compared with the saline group, including IL-1 $\alpha$, IL-1 $\beta$, IL-6, IL-12 and IL-13, implying that inflammation may have a vital role in the development of LPS-induced BPD. Numerous studies have provided epidemiological data indicating a strong correlation between the exposure in utero to chorioamnionitis and the occurrence of 'new BPD'. High concentrations of pro-inflammatory cytokines in human amniotic fluid have been identified as one of risk factors for $\operatorname{BPD}(2,12,13,14)$. A number of studies also demonstrated that
Table III. Cytokine expression downregulated by $\geq 2$-fold in the LPS+theo group compared with the LPS group.

\begin{tabular}{lccr}
\hline Cytokine & LPS+theo & LPS & Fold change \\
\hline TNF- $\alpha$ & 0.0036945 & 0.1380842 & 37.3756124 \\
EGFR & 0.0077487 & 0.1818090 & 23.4631616 \\
VEGF & 0.0089257 & 0.1235228 & 14.9920866 \\
MIP-1 $\alpha$ & 0.0082392 & 0.1033795 & 12.5472740 \\
B7-1/CD80 & 0.0102989 & 0.1287002 & 12.4964996 \\
CINC-2 $\alpha / \beta$ & 0.0105605 & 0.1288620 & 12.2022631 \\
RALT/MIG-6 & 0.0428305 & 0.3737368 & 8.7259500 \\
MIP-2 & 0.0068333 & 0.0589671 & 8.6293738 \\
RAGE & 0.0547642 & 0.3713908 & 6.7816347 \\
CXCR4 & 0.0402149 & 0.2192642 & 5.4523125 \\
Prolactin R & 0.0683000 & 0.3356344 & 4.9141201 \\
CD106 & 0.0651285 & 0.2237945 & 3.4361992 \\
RELM $\beta$ & 0.1340824 & 0.4076326 & 3.0401649 \\
CSK & 0.0662075 & 0.1999703 & 3.0203572 \\
FGF-BP & 0.0609436 & 0.1841550 & 3.0217283 \\
VEGF-C & 0.0142550 & 0.0328374 & 2.3035707
\end{tabular}

TNF- $\alpha$, tumor necrosis factor- $\alpha$; EGFR, epidermal growth factor receptor; VEGF, vascular endothelial growth factor; MIP, macrophage inflammatory protein; CINC-2 $\alpha / \beta$, CINC, cytokine-induced neutrophil chemoattractant- $\alpha / \beta$; RALT/MIG, receptor-associated late transducer/mitogen inducible gene-6; RAGE, receptor for advanced glycation endproducts CXCR4, C-X-C chemokine receptor type 4; RELM $\beta$, resistin-like molecule $\beta$; CSK, c-src tyrosine kinase; FGF-BP, fibroblast growth factor-binding protein; VEGF-C, vascular endothelial growth factor-C.

intra-amnionitic injection of a high dose of endotoxin may induce more pronounced expression of cytokines and notably longer existence of inflammatory cells in the lungs than in chorioamnionitis $(2,15,16,17)$. Premature infants at various stages of the development of BPD have notably higher and persisting numbers of neutrophils and macrophages, both of which have essential roles in pulmonary inflammatory responses $(18,19,20)$. Furthermore, elevated protein and mRNA levels of certain pro-inflammatory cytokines and chemokines have also been detected in the airway secretions and bronchoalveolar cells of infants with developing BPD; however, cellular mRNA for IL-10, a classical antiinflammatory cytokine, was undetectable in the majority of airway samples (21). This evidence suggests that BPD results, at least in part, from a persistent imbalance between proinflammatory and anti-inflammatory mechanisms, which favors pro-inflammatory processes.

Theophylline was initially used in the treatment of respiratory diseases, including bronchial asthma and COPD, attributed to its beneficial effects on bronchodilation and anti-inflammatory activity $(22,23)$. Consensus is held that theophylline at a high dose inhibits PDEs, synergizes with the activators of adenylatecyclase, antagonizes adenosine receptors, increases cellular cAMP concentration and inhibits $\mathrm{Ca}^{2+}$ influxes into the cell, relaxing airway smooth muscle $(6,22,24)$. However, the reported frequent side effects 
associated with high therapeutic doses limit its clinical use. Furthermore, to date, the anti-inflammatory mechanisms of theophylline at physiological concentrations remain ambiguous. The proposed mechanisms vary from inducing IL-10 release to mediator inhibition or increased apoptosis of inflammatory cells $(22,24,25)$. In recent years, a considerable number of studies have indicated that it is more likely that the transcription-dependent mechanism at relative low dose-physiological concentrations, by inhibition of PI3K, inhibition of transcription factor NF- $\mathrm{KB}$ and induction of HDAC activity, perhaps with histone acetylation/deacetylation as the point of convergence, is involved in preventing the transcription of pro-inflammatory cytokines (26). Most importantly, the minimal detrimental effects associated with physiological doses improve the clinical applications of theophylline and provide more choices for BPD therapy.

To improve the understanding of the effect of theophylline on the inflammatory regulation in developing BPD induced by chorioamnionitis, a cytokine antibody array for the multiplex analysis of 90 rat cytokines was conducted to detect the differential expression pattern between the LPS+theo and LPS groups. Utilizing high-throughput screening of the protein expression levels, it was identified that the inflammatory cytokines TNF- $\alpha$, MIP-1 $\alpha$ and MIP-2 had a similar decreasing trend in the LPS+theo group compared with the LPS group. The anti-inflammatory cytokine TGF- $\beta$ family members TGF- $\beta 1$, TGF- $\beta 2$ and TGF- $\beta 3$ increased markedly, particularly TGF- $\beta 2$ and TGF- $\beta 3$. As a result, the cytokine profiling confirmed the hypothesis that theophylline ameliorates the inflammatory cytokine imbalance in BPD of neonatal rats induced by chorioamnionitis.

In addition to the effect of theophylline in directly suppressing the expression of inflammatory cytokines, previous studies have reported that theophylline may also increase the production of IL-10, a well-established cytokine to indirectly downregulate the release of several pro-inflammatory cytokines, including as TNF- $\alpha$, IL-1 $\beta$, IL-6, IL-8, MIP-1 $\alpha$ and granulocyte macrophage-colony stimulating factor from monocytes and alveolar macrophages $(5,27)$. The cytokine profiling of the antibody array of the present study indicated that, in this BPD model, theophylline upregulated the expression levels of another anti-inflammatory cytokine, TGF- $\beta$, but not IL-10, which is a result that, to the best of our knowledge, has not been previously reported. It is well established that TGF- $\beta$ and IL-10 are Th3 cytokines and thus exert immunosuppressive actions. TGF- $\beta$ is not only a regulator of cell proliferation, differentiation and migration, but also an important immunomodulator (28). Previous studies have reported that TGF- $\beta 1$-deficient mice spontaneously developed autoimmune diseases with uncontrollable systemic inflammation and died in utero or perinatally due to widespread inflammation (29). Another study revealed a protective role of TGF- $\beta$ signaling in nuclear factor (NF)- $\kappa B$-mediated acute peritonitis. The upregulation of endogenous peritoneal TGF- $\beta 1$ and the activation of TGF- $\beta$ signaling were closely associated with a significant inhibition of NF- $\mathrm{\kappa B}$ activation and mitigation of acute peritoneal inflammation following E. coli infection (30). Kitamura et al (31) demonstrated that cAMP induced the secretion 10 -fold of bioactive TGF- $\beta 2$ in PC-3 cells. Therefore, it is hypothesized that theophylline may increase cellular cAMP levels by PDE inhibition and adenosine receptor antagonism to induce bioactive TGF- $\beta 2$ at the level of either transcription or messenger stability.

In conclusion, theophylline may restore the balance between pro-and anti-inflammatory mechanisms by downregulating the expression of the pro-inflammatory cytokines, TGF- $\alpha$, MIP- $1 \alpha$ and MIP-2 and upregulating anti-inflammatory cytokines including TGF- $\beta$ family members to improve alveolarization in BPD of neonatal rats induced by chorioamnionitis. Furthermore, other categories of cytokines in the arrays, including growth factor signaling protein nerve growth factor receptor, basic fibroblast growth factor, cell adhesion and motility related protein E-Selectin, C-src tyrosine kinase and cell apoptosis related protein FADD, were also altered by $>2$-fold, which may contribute to the arrested alveolar development in BPD. Thus, we propose that theophylline may ameliorate alveolarization disruption of BPD through regulating the balance between pro- and anti-inflammatory mechanisms, which provides a novel indication of clinical therapy for BPD.

\section{Acknowledgements}

The present study was supported by the Shanghai Natural Science Foundation (grant no. 11ZR1423800) and National Natural Science Foundation of China (grant no. 81270729) (to Y. Zhang).

\section{References}

1. Choi CW, Kim BI, Hong JS, Kim EK, Kim HS and Choi JH: Bronchopulmonary dysplasia in a rat model induced by intra-amniotic inflammation and postnatal hyperoxia: morphometric aspects. Pediatr Res 65: 323-327, 2009.

2. Speer CP: Inflammation and bronchopulmonary dysplasia: A continuing story. Semin Fetal Neonatal Med 11: 354-362, 2006.

3. Jarreau PH, Fayon M, Baud O, Autret-Leca E, Danan M, de Verdelhan A and Castot A: The use of postnatal corticosteroid therapy in premature infants to prevent or treat bronchopulmonary dysplasia: current situation and recommendations. Arch Pediatr 17: 1480-1487, 2010 (In French).

4. Grier DG and Halliday HL: Corticosteroids in the prevention and management of bronchopulmonary dysplasia. Semin Neonatol 8: 83-91, 2003.

5. To Y, Ito K, Kizawa Y, Failla M, Ito M, Kusama T, Elliott WM, Hogg JC, Adcock IM and Barnes PJ: Targeting phosphoinositide-3-kinase-delta with theophylline reverses corticosteroid insensitivity in chronic obstructive pulmonary disease. Am J Respir Crit Care Med 182: 897-904, 2010.

6. Mascali JJ, Cvietusa P, Negri J and Borish L: Anti-inflammatory effects of theophylline: modulation of cytokine production. Ann Allergy Asthma Immunol 77: 34-38, 1996.

7. Zhu L, Li H, Tang J, Zhu J and Zhang Y: Hyperoxia arrests alveolar development through suppression of histone deacetylases in neonatal rats. Pediatr Pulmonol 47: 264-274, 2012.

8. Coalson JJ: Pathology of bronchopulmonary dysplasia. Semin Perinatol 30: 179-184, 2006.

9. Schreiber T, Niemann C, Schmidt B and Karzai W: A novel model of selective lung ventilation to investigate the long-term effects of ventilation-induced lung injury. Shock 26: 50-54, 2006.

10. Kramer EL, Deutsch GH, Sartor MA, Hardie WD, Ikegami M, Korfhagen TR and Le Cras TD: Perinatal increases in TGF- $\alpha$ disrupt the saccular phase of lung morphogenesis and cause remodeling: microarray analysis. Am J Physiol Lung Cell Mol Physiol 293: L314-L327, 2007.

11. Kim N and Vu TH: Parabronchial smooth muscle cells and alveolar myofibroblasts in lung development. Birth Defects Res C Embryo Today 78: 80-89, 2006.

12. Bland RD: Neonatal chronic lung disease in the post-surfactant era. Biol Neonate 88: 181-191, 2005. 
13. Vohr BR, Wright LL, Dusick AM, et al: Neurodevelopmental and functional outcomes of extremely low birth weight infants in the National Institute of Child Health and Human Development Neonatal Research Network, 1993-1994. Pediatrics 105: 1216-1226, 2000.

14. Yoon BH, Romero R, Jun JK, et al: Amniotic fluid cytokines (interleukin-6, tumor necrosis factor-alpha, interleukin-1 beta, and interleukin-8) and the risk for the development of bronchopulmonary dysplasia. Am J Obstet Gynecol 177: 825-830, 1997.

15. Speer CP, Pabst MJ, Hedegaard HB, Rest RF and Johnston RB Jr: Enhanced release of oxygen metabolites by monocyte-derived macrophages exposed to proteolytic enzymes: activity of neutrophi elastase and cathepsin G. J Immunol 133: 2151-2156, 1984.

16. Yoon BH, Romero R, Jun JK, et al: Amniotic fluid cytokines (interleukin-6, tumor necrosis factor-alpha, interleukin-1 beta, and interleukin-8) and the risk for the development of bronchopulmonary dysplasia. Am J Obstet Gynecol 177: 825-830, 1997.

17. Gomez R, Romero R, Ghezzi F, Yoon BH, Mazor M and Berry SM: The fetal inflammatory response syndrome. Am J Obstet Gynecol 179: 194-202, 1998.

18. Arnon S, Grigg J and Silverman M: Pulmonary inflammatory cells in ventilated preterm infants: effect of surfactant treatment. Arch Dis Child 69: 44-48, 1993.

19. Kotecha S, Chan B, Azam N, Silverman M and Shaw RJ: Increase in interleukin-8 and soluble intercellular adhesion molecule-1 in bronchoalveolar lavage of premature infants with chronic lung disease. Arch Dis Child Fetal Neonatal Ed 72: F90-F96, 1995.

20. Kotecha S, Mildner RJ, Prince LR, et al: The role of neutrophil apoptosis in the resolution of acute lung injury in newborn infants Thorax 58: 961-967, 2003.

21. Jones CA, Cayabyab RG, Kwong KY, et al: Undetectable interleukin (IL)-10 and persistent IL-8 expression early in hyaline membrane disease: a possible developmental basis for the predisposition to chronic lung inflammation in preterm newborns. Pediatr Res 39: 966-975, 1996
22. Kaneko Y, Takashima K, Suzuki N and Yamana K: Effects of theophylline on chronic inflammatory lung injury induced by LPS exposure in guinea pigs. Allergol Int 56: 445-456, 2007.

23. Ito K, Lim S, Caramori G, et al: A molecular mechanism of action of theophylline: Induction of histone deacetylase activity to decrease inflammatory gene expression. Proc Natl Acad Sci USA 99: 8921-8926, 2002.

24. Liang B, Jiang S, Zhang Z, et al: Anti-inflammatory effects of theophylline: modulation of immune functions during murine leukemia virus infection. Immunopharmacol Immunotoxicol 23: 307-319, 2001.

25. Ezeamuzie CI and Shihab PK: Interactions between theophylline and salbutamol on cytokine release in human monocytes. J Pharmacol Exp Ther 334: 302-309, 2010.

26. Marwick JA, Caramori G, Stevenson CS, et al: Inhibition of PI3Kdelta restores glucocorticoid function in smoking-induced airway inflammation in mice. Am J Respir Crit Care Med 179: 542-548, 2009.

27. Bonner JC: The epidermal growth factor receptor at the crossroads of airway remodeling. Am J Physiol Lung Cell Mol Physiol 283: L528-L530, 2002.

28. Lutgens E, Gijbels M, Smook M, Heeringa P, Gotwals P, Koteliansky VE and Daemen MJ: Transforming growth factor-beta mediates balance between inflammation and fibrosis during plaque progression. Arterioscler Thromb Vasc Biol 22: 975-982, 2002.

29. Shull MM, Ormsby I, Kier AB, et al: Targeted disruption of the mouse transforming growth factor-beta 1 gene results in multifocal inflammatory disease. Nature 359: 693-699, 1992.

30. Lan HY: Diverse roles of TGF- $\beta /$ Smads in renal fibrosis and inflammation. Int J Biol Sci 7: 1056-1067, 2011.

31. Kitamura H, Cambier S, Somanath S, et al: Mouse and human lung fibroblasts regulate dendritic cell trafficking, airway inflammation, and fibrosis through integrin $\alpha v \beta 8$-mediated activation of TGF- $\beta$. J Clin Invest 121: 2863-2875, 2011. 